\title{
Developmental Physiology: Grand Challenges
}

\begin{abstract}
Warren Burggren *
Developmental Integrative Biology Research Group, Department of Biological Sciences, University of North Texas, Denton, TX, United States
\end{abstract}

Keywords: DOHaD, developmental plasticity, climate change, toxicology, epigenetics, heterokairy, allometry, animal models

\section{INTRODUCTION}

The field of Developmental Physiology faces both great opportunities and significant challenges in the years ahead. While this provides "job security" for new investigators in this area, it also requires delineating some of the major challenges to maximize progress. I suggest that pressing challenges in Developmental Physiology fall into three categories: demonstrating broad relevance, promoting conceptual advances and improving experimental approaches.

\section{CHALLENGE: DEMONSTRATING BROAD RELEVANCE Developmental Origins of Health and Disease}

The developmental origins of health and disease (DOHaD) encapsulates how environmental stressors in early development can lead to subsequent health implications in adult humans (Figure 1). DOHaD is now influencing many fields of research (Suzuki, 2018), including epidemiology (Heindel et al., 2017), epigenetics (Safi-Stibler and Gabory, 2020), specific pathophysiologies (Arima and Fukuoka, 2020; Briana and Malamitsi-Puchner, 2020) and policy and public health (Loi et al., 2013), to name but a few such fields. Among the many challenges for human-oriented studies in $\mathrm{DOHaD}$ is translating what we learn from developmental studies into actionable clinical and public health practices (Hanson et al., 2019). What is increasingly understood is how early developmental trajectories can be programmed by early developmental events. Yet, adult disease prevention through intervention in early development in many instances lags behind the more common (and far more challenging, not to mention expensive) practice of treating diseases in adults arising from, as but one example, maternal or neonatal nutrition (Baird et al., 2017). In basic animal physiology, the consequences of early life conditions for adult experimental animals are not generally appreciated (or are ignored), judging from the consistent lack of any mention of rearing conditions in published physiology articles using adult animal models. Recognizing how early life conditions alter adult physiology remains a challenge for the field.

\section{Developmental Physiology and Climate Change}

Great effort is being expended to determine the effects of projected climate change on individual organisms all the way up to the ecosystems they inhabit. All too often, however, studies singularly involve adults of a species. Far less studied are effects in developing animals of temperature change, ocean acidification and other climate change-related phenomena. This exposes a truism: there will be no adults of a species to experience climate change effects if the species' offspring do not develop correctly (or at all) in the first place! Indeed, climate change presents numerous possible stressors that could affect development in a similar manner to DOHaD-related stressors during the developmental process (Kingsolver and Buckley, 2020; Sanger, 2021). One of the challenges for 


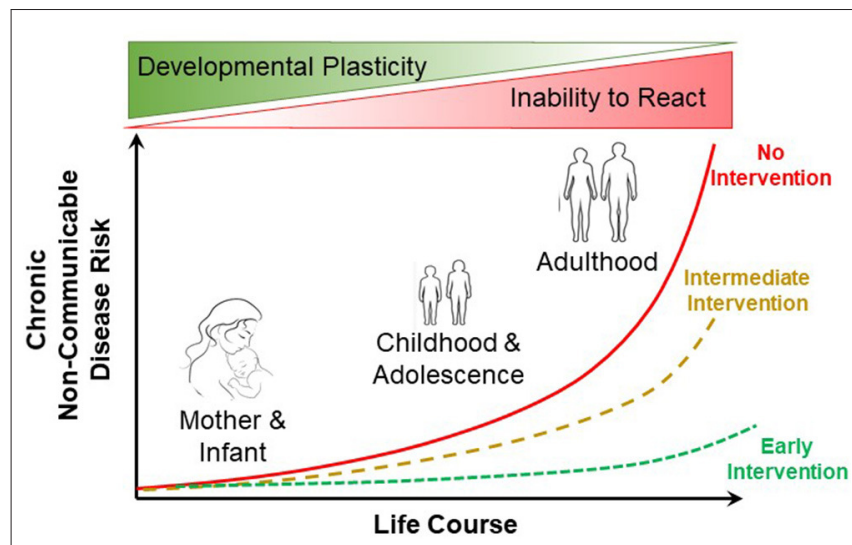

FIGURE 1 | Influence of early life course intervention on the risk of non-communicable disease in humans. Early intervention (e.g., improved maternal and neonatal nutrition) can result in greatly reduced risk of non-communicable disease in adulthood. Modified from Baird et al. (2017).



FIGURE 2 | Heterochrony compared with heterokairy. (A) Heterochrony compares the timing of developmental landmark between a species of interest and the ancestral species. (B) In contrast, heterokairy compares the developmental landmarks of two different individuals or populations within a single species. Typically, the developmental landmarks are anatomical-e.g., the appearance of gill arches, cardiac valves or nephrons. However, these concepts apply equally well to physiological developmental landmarks such as the onset of gill ventilation, the onset of anterograde blood flow through the heart, or the first production of urine.

Developmental Physiology, then, is to emphasize and analyze effects of climate change on early life stages and how this ultimately influences species fitness.

\section{A Deeper Understanding of Toxicological Effects During Development}

The early life stage approach advocated above has been exploited in toxicology and the study of environmental contamination. A prime example of this is the April 2010 DeepWater Horizon oil spill in the Gulf of Mexico, where considerable focus has been on the effects of oil exposure in developing fish and birds. What emerges is that early life stages have often higher vulnerability to environmental toxicants than at later stages (Incardona and Scholz, 2018; Pasparakis et al., 2019). Yet, what is also becoming clear is that organisms can develop considerable resilience to toxicants that can transfer to their offspring through non-genetic mechanisms (Vandegehuchte and Janssen, 2014; Kishimoto et al., 2017; Bautista and Burggren, 2019). A challenge, then is to understand the limits and mechanisms underlying inheritance of this toxicant resistance and how this might alter both science and policy involving environmental contamination.

\section{CHALLENGE: PROMOTING CONCEPTUAL ADVANCES}

\section{Expanding the Role of Epigenetics}

Mendelian genetics is increasingly inadequate in explaining key developmental phenomena ranging from misdirected gene expression resulting in adult disease states to non-genetically inheritance of modified phenotypes. We now know that, typically, the modification of DNA expression in germ or somatic cells alike that produces modified phenotypes involves "readers" that assess the state of epigenetic markers on genes. Subsequently, "writers" and "erasers" modify the state of epigenetic markers according to the prevailing environment and thus modify expression of genes that these markers regulate. The result is altered phenotypes within or across generations (Walker and Burggren, 2020; Wan et al., 2021; Wei and He, 2021). Essentially, developmental phenotypic plasticity, one of the basic principles of developmental physiology (Burggren, 2020a) and discussed above in the context of DOHaD, derives largely from the writing and erasing of epigenetic markers. A challenge for developmental physiologists is to integrate, from molecular to organismal level, the mechanisms and outcomes associated with epigenetic reading, writing and erasing and the associated changes in gene expression, both within and across generations.

\section{Physiological Heterochrony and Heterokairy}

Heterochrony is the change in timing of developmental landmarks in a species compared to its ancestral species (Keyte and Smith, 2014). As a companion concept, heterokairy focuses on the intraspecific change in timing of developmental landmarks in an individual or a population (Figure 2). Like heterochrony, the concept of heterokairy is proving similarly useful in understanding developmental processes (Spicer and Burggren, 2003; Rundle and Spicer, 2016; Spicer et al., 2018). However, both of these concepts are typically employed to consider anatomical development with typically less focus on physiological processes. Yet, both concepts also equally apply to physiological development. The challenge for developmental physiologists, then, is to more deeply investigate 
heterochrony and especially heterokairy, allowing important insights into phenomena such as developmental phenotypic plasticity and the contributions of physiological processes to fitness during development.

\section{Allometry and Development}

The allometry of development has long been of interest to developmental physiologists (Gould, 1975; Weder and Schork, 1994; Stern and Emlen, 1999; Singer and Mühlfeld, 2007; Vea and Shingleton, 2021). However, a fundamental challenge that remains unresolved involves reconciling two basic yet conflicting tenets of allometry and development (Burggren, 2020b). Thus, a basic tenet of allometry is that animals of different sizes being compared should be in the same physiological state. Yet, a basic tenet of development is that developing animals are constantly changing physiological state. How then, can allometric analyses simplistically involve both a larva or fetus as well an adult? This is not an insurmountable problem, as there are likely to emerge (or, at least should emerge) weighted statistical approaches that take changing physiology into account as allometric analyses are performed (or vice versa).

\section{CHALLENGE: IMPROVING EXPERIMENTAL APPROACHES}

\section{Seeing Development as a Continuum Rather Than Discrete Events}

Traditionally, developmental physiology has been built upon individual studies carried out at a single or just a few discrete points in development. Yet, I posit that the most robust understanding of "how physiology develops" lies in considering a continuum from germ cells to organismal senescence (as encapsulated in the description of the Developmental Physiology Section of Frontiers in Physiology). Any attempted synthesis of developmental physiology gleaned from analysis of multiple physiological studies each conducted at only a single point of development will, at best, be tedious and, at worst, lead to a biased or inaccurate conclusion. By considering a continuum, physiological measurements can more readily be put in context of an organism's entire life cycle. Thus, a specific challenge is to promote experimental protocols that gather data along multiple points of the developmental continuum.

\section{Expanding (and Verifying) our Animal Models}

The use of animal models is, of course, a stalwart of developmental physiological investigation. Whether the larvae of zebrafish or Xenopus, the chicken embryo, or the mouse or sheep fetus, animal models have been of major benefit to both enhance our basic understanding of the "biology of development" as well as provide information of translational importance to biomedical research. Additionally, invertebrate models including the fruit fly Drosophila melanogaster and the nematode Caenorhabditis elegans have, as animal models, provided great insight into development. Yet, the challenge is to not become complacent in our use of these animal models. As the Danish physiologist and Nobel Prize winner August Krogh declared, for every physiological question there is the ideal animal to answer it (Krogh, 1929). Initially successful animal models become entrenched in the research community, sometimes resulting in the lack of penetration or marginalization of alternative animal models that could prove to be superior in some respects (Flores Santin and Burggren, submitted).

\section{Experiments Incorporating Multiple Stressor Experiments and Stochasticity}

By the nature of our training, physiologists prefer to hold constant all but a single dependent environmental variable of interest (e.g., temperature, $\mathrm{pH}$, oxygen), which is then controlled or allowed to vary. Yet, in the "real world," of course, multiple variables are constantly changing concurrently. Thus, aquatic environments may be hypoxic because they are also warm, while high altitude terrestrial environments may carry have low oxygen and high radiation loads combined with low temperatures and humidities. In recognition of this, many developmental physiologists are beginning to expose developing animals to multiple rather than single changing stressors (Figure 3).

What is emerging from "multiple variable experiments" is that different organ systems and their system-specific physiologies have different critical windows for development. A challenge is to generate enhanced understanding of the interaction of development, time and environment through multi-variable experiments (Mueller, 2018).

\section{Riding the Wave of Technological Innovation}

Developing animals are almost inevitably small, if not microscopic. Not surprisingly, then, miniaturization has long been a priority in instrumentation for developmental physiology (Burggren and Fritsche, 1995). Several recent technological developments have been aiding the assessment of physiological processes in progressively smaller (and thus earlier) developmental stages. Advances in imaging continues to improve our understanding of especial cardiac physiology of embryos, fetuses and larvae (Aguet et al., 2020; Salman and Yalcin, 2020; Lopez and Larina, 2021). The advent of microfluidics is contributing to our knowledge of embryonic physiology, especially as it pertains to in vitro embryo production (Wheeler and Rubessa, 2017; Sonnen and Merten, 2019). Organoids ("organs on a chip") are increasingly being studied either on their own or as components of increasingly complex "organ" systems (Matsui et al., 2021). The study of organoids may yield extraordinary new insights into the assembly of the cells and tissues in early development. These and other emerging techniques typically produce vast amounts of data, often in the form of complex images. Not surprisingly, then, developmental biology, including developmental physiology, has begun to exploit to machine ("deep") learning, which can analyze large data sets without direct human involvement and the associated 



FIGURE 3 | Multi-variable experimental designs and possible outcomes. (A) A three-dimensional schematic depiction of a protocol that would employ multiple levels/concentrations/doses of multiple variables. (B) A hypothetical outcome on a developing animals phenotype produced by simultaneous variation in two different variables. In this schematic, the largest effect is created by a combination of intermediate levels Variable \#1 and Variable \#2. Modified from Burggren and Mueller (2015). (C) An actual example of interactions between development (Variable \#1) and rearing salinity (Variable \#2) in the brine shrimp Artemia franciscana. Note that there was an unexpected bimodal effect of rearing salinity, with the largest effect appearing early in development. Modified from Mueller et al. (2016).

risk of human-created error (Feltes et al., 2018; Villoutreix, 2021).

There are challenges associated with these and numerous other emerging technological innovations in the engineering processes, which continue to inexorably advance. Yet, the greater challenge may be providing broad access to these technologies and the training required to deploy them.

\section{CONCLUSIONS}

In this short perspective on Grand Challenges in Developmental Physiology I have indicated directions in which the field of Developmental Physiology might (not necessarily should) move. Some of my predictions and suggestions will be on target, others less important than predicted, and new unimagined Challenges will emerged. What is crucial is that Developmental Physiology does not become complacent regarding its many achievements, continues to thrive on collaboration with other physiological disciplines, and ensures that its trainees are moving to the next stages of their careers with the skills and enthusiasm that continues to be a hallmark of developmental physiologists.

\section{AUTHOR CONTRIBUTIONS}

The author confirms being the sole contributor of this work and has approved it for publication.

\section{ACKNOWLEDGMENTS}

I thank the many colleagues and students (and students who have become colleagues) who, through animated discussions over the years, have provided me with most of the ideas outlined in this brief article. 


\section{REFERENCES}

Aguet, J., Seed, M., and Marini, D. (2020). Fetal cardiovascular magnetic resonance imaging. Pediatr. Radiol. 50, 1881-1894. doi: 10.1007/s00247-02004902-y

Arima, Y., and Fukuoka, H. (2020). Developmental origins of health and disease theory in cardiology. J. Cardiol. 76, 14-17. doi: 10.1016/j.jjcc.2020.02.003

Baird, J., Jacob, C., Barker, M., Fall, C. H., Hanson, M., Harvey, N. C., et al. (2017). Developmental origins of health and disease: a lifecourse approach to the prevention of non-communicable diseases. Healthcare 5:14. doi: 10.3390/healthcare5010014

Bautista, N. M., and Burggren, W. W. (2019). Parental stressor exposure simultaneously conveys both adaptive and maladaptive larval phenotypes through epigenetic inheritance in the zebrafish (Danio rerio). J Exp Biol. 222:208918. doi: $10.1242 /$ jeb.208918

Briana, D. D., and Malamitsi-Puchner, A. (2020). Perinatal biomarkers implying 'developmental origins of health and disease' consequences in intrauterine growth restriction. Acta Paediatr. 109, 1317-1322. doi: 10.1111/apa.15022

Burggren, W., and Fritsche, R. (1995). Cardiovascular measurements in animals in the milligram range. Braz. J. Med. Biol. Res. 28, 1291-1305.

Burggren, W. W. (2020a). Phenotypic switching resulting from develpomental plasticity: fixed or reversible? Front. Physiol. 10:1634. doi: $10.3389 /$ fphys.2019.01634

Burggren, W. W. (2020b). Putting the August Krogh principle to work in developmental physiology. Comp. Biochem. Physiol. A Mol Integr. Physiol. 252:110825. doi: 10.1016/j.cbpa.2020.110825

Burggren, W. W., and Mueller, C. A. (2015). Developmental critical windows and sensitive periods as three-dimensional constructs in time and space. Physiol. Biochem. Zool. 88, 91-102. doi: 10.1086/679906

Feltes, B. C., Grisci, B. I., Poloni, J. F., and Dorn, M. (2018). Perspectives and applications of machine learning for evolutionary developmental biology. Mol. Omics 14, 289-306. doi: 10.1039/C8MO00111A

Gould, S. J. (1975). Allometry in primates, with emphasis on scaling and the evolution of the brain. Contrib. Primatol. 5, 244-292.

Hanson, M. A., Poston, L., and Gluckman, P. D. (2019). DOHaD - the challenge of translating the science to policy. J. Dev. Orig. Health Dis. 10, 263-267. doi: $10.1017 /$ S2040174419000205

Heindel, J. J., Skalla, L. A., Joubert, B. R., Dilworth, C. H., and Gray, K. A. (2017). Review of developmental origins of health and disease publications in environmental epidemiology. Reprod. Toxicol. 68, 34-48. doi: $10.1016 /$ j.reprotox.2016.11.011

Incardona, J. P., and Scholz, N. L. (2018). "Case study: the 2010 deepwater horizon oil spill and its environmental developmental impacts," in Development and Environment, eds W. W. Burggren, and B. Dubansky (Cham: Springer), 253-283. doi: 10.1007/978-3-319-75935-7_10

Keyte, A. L., and Smith, K. K. (2014). Heterochrony and developmental timing mechanisms: changing ontogenies in evolution. Semin. Cell Dev. Biol. 34, 99-107. doi: 10.1016/j.semcdb.2014.06.015

Kingsolver, J. G., and Buckley, L. B. (2020). Ontogenetic variation in thermal sensitivity shapes insect ecological responses to climate change. Curr. Opin. Insect Sci. 41, 17-24. doi: 10.1016/j.cois.2020.05.005

Kishimoto, S., Uno, M., Okabe, E., Nono, M., and Nishida, E. (2017). Environmental stresses induce transgenerationally inheritable survival advantages via germline-to-soma communication in Caenorhabditis elegans. Nat. Commun. 8:14031. doi: 10.1038/ncomms14031

Krogh, A. (1929). The progress of physiology. Am. J. Physiol. 90, 243-251. doi: 10.1152/ajplegacy.1929.90.2.243

Loi, M., Del Savio, L., and Stupka, E. (2013). Social epigenetics and equality of opportunity. Public Health Ethics 6, 142-153. doi: 10.1093/phe/pht019

Lopez, A. L. III., and Larina, I. V. (2021). Dynamic imaging of mouse embryos and cardiac development in static culture. Methods Mol. Biol. 2206, 129-141. doi: 10.1007/978-1-0716-0916-3_10

Matsui, T. K., Tsuru, Y., Hasegawa, K., and Kuwako, K. I. (2021). Vascularization of human brain organoids. Stem Cells. doi: 10.1002/stem.3368. [Epub ahead of print].

Mueller, C. (2018). "Critical windows in animal development: interactions between environment, phenotype, and time," in Development and Environment, eds W. W. Burggren, and B. Dubansky (Cham: Springer), 41-72. doi: 10.1007/978-3-319-75935-7_3
Mueller, C. A., Willis, C., and Burggren, W. W. (2016). Salt sensitivity of the morphometry of Artemia franciscana during development: a demonstration of 3-D critical windows. J Exp Biol 219, 571-581. doi: 10.1242/jeb. 125823

Pasparakis, C., Grosell, M., Esbaugh, A., and Burggren, W. W. (2019). Physiological effects of deepwater horizon oil on fish. Comp. Physiol. Biochem. 224:108558. doi: 10.1016/j.cbpc.2019.06.002

Rundle, S. D., and Spicer, J. I. (2016). Heterokairy: a significant form of developmental plasticity? Biol. Lett. 12:20160509. doi: 10.1098/rsbl.2016.0509

Safi-Stibler, S., and Gabory, A. (2020). Epigenetics and the developmental origins of health and disease: parental environment signalling to the epigenome, critical time windows and sculpting the adult phenotype. Semin. Cell Dev. Biol. 97, 172-180. doi: $10.1016 /$ j.semcdb.2019.09.008

Salman, H. E., and Yalcin, H. C. (2020). Advanced blood flow assessment in Zebrafish via experimental digital particle image velocimetry and computational fluid dynamics modeling. Micron 130:102801. doi: 10.1016/j.micron.2019.102801

Sanger, T. J. (2021). Integrative developmental biology in the age of anthropogenic change. Evol. Dev. e12377. doi: 10.1111/ede.12377. [Epub ahead of print].

Singer, D., and Mühlfeld, C. (2007). Perinatal adaptation in mammals: the impact of metabolic rate. Comp. Biochem. Physiol. A Mol. Integr. Physiol. 148, 780-784. doi: 10.1016/j.cbpa.2007.05.004

Sonnen, K. F., and Merten, C. A. (2019). Microfluidics as an emerging precision tool in developmental biology. Dev. Cell. 48, 293-311. doi: 10.1016/j.devcel.2019.01.015

Spicer, J. I., and Burggren, W. W. (2003). Development of physiological regulatory systems: altering the timing of crucial events. Zoology 106, 91-99. doi: 10.1078/0944-2006-00103

Spicer, J. I., Tills, O., Truebano, M., and Rundle, S. D. (2018). "Developmental plasticity and heterokairy," in Develpment and Environment, eds W. W. Burggren, and B. Dubansky (Cham: Springer), 73-96. doi: 10.1007/978-3-319-75935-7_4

Stern, D. L., and Emlen, D. J. (1999). The developmental basis for allometry in insects. Development 126, 1091-1101. doi: 10.1242/dev.126.6.1091

Suzuki, K. (2018). The developing world of DOHaD. J. Dev. Orig. Health Dis. 9, 266-269. doi: 10.1017/S2040174417000691

Vandegehuchte, M. B., and Janssen, C. R. (2014). Epigenetics in an ecotoxicological context. Mutat. Res. Genet. Toxicol. Environ. Mutagen. 764-765, 36-45. doi: 10.1016/j.mrgentox.2013.08.008

Vea, I. M., and Shingleton, A. W. (2021). Network-regulated organ allometry: the developmental regulation of morphological scaling. Wiley Interdiscip. Rev. Dev. Biol. 10:e391. doi: 10.1002/wdev.391

Villoutreix, P. (2021). What machine learning can do for developmental biology. Development 148:dev188474. doi: 10.1242/dev.188474

Walker, C., and Burggren, W. W. (2020). Remodeling the epigenome and (epi)cytoskeleton: a new paradigm for co-regulation by methylation. J. Exp. Biol. 223:jeb220632. doi: 10.1242/jeb.220632

Wan, C., Zhang, F., Yao, H., Li, H., and Tuan, R. S. (2021). Histone modifications and chondrocyte fate: regulation and therapeutic implications. Front. Cell Dev. Biol. 9:626708. doi: 10.3389/fcell.2021.626708

Weder, A. B., and Schork, N. J. (1994). Adaptation, allometry, and hypertension. Hypertension 24, 145-156. doi: 10.1161/01.HYP.24.2.145

Wei, J., and He, C. (2021). Chromatin and transcriptional regulation by reversible RNA methylation. Curr. Opin. Cell Biol. 70, 109-115. doi: 10.1016/j.ceb.2020.11.005

Wheeler, M. B., and Rubessa, M. (2017). Integration of microfluidics in animal in vitro embryo production. Mol. Hum. Reprod. 23, 248-256. doi: 10.1093/molehr/gax009

Conflict of Interest: The author declares that the research was conducted in the absence of any commercial or financial relationships that could be construed as a potential conflict of interest.

Copyright $\odot 2021$ Burggren. This is an open-access article distributed under the terms of the Creative Commons Attribution License (CC BY). The use, distribution or reproduction in other forums is permitted, provided the original author $(s)$ and the copyright owner(s) are credited and that the original publication in this journal is cited, in accordance with accepted academic practice. No use, distribution or reproduction is permitted which does not comply with these terms. 